\title{
Dark gas: a new possible link between low and high-energy phenomena
}

\section{Kazufumi Torii ${ }^{1}$, Yasuo Fukui ${ }^{1}$, Hidetoshi Sano ${ }^{1}$, Junki Sato ${ }^{1}$, Takeshi Okuda $^{1}$, Hiroaki Yamamoto ${ }^{1}$, Akiko Kawamura ${ }^{2}$, Norikazu Mizuno $^{2}$, Toshikazu Onishi ${ }^{3}$ and Hideo Ogawa ${ }^{3}$}

\author{
${ }^{1}$ Department of Physics and Astrophysics, Nagoya University, Furo-cho, Chikusa-ku, Nagoya, \\ Aichi 464-8601, Japan \\ ${ }^{2}$ National Astronomical Observatory of Japan, Mitaka, Tokyo 181-8588, Japan \\ ${ }^{3}$ Department of Astrophysics, Graduate School of Science, Osaka Prefecture University, 1-1 \\ Gakuen-cho, Naka-ku, Sakai, Osaka 599-8531, Japan
}

email: torii@a.phys.nagoya-u.ac.jp

\begin{abstract}
Galactic-scale studies of $\gamma$-rays and sub-mm radiation suggest that a significant amount of neutral interstellar medium is not detectable either in CO or HI (Grenier et al. 2005; Ade et al. 2011). This component is called "dark gas". Here we argue that cool and dense atomic gas without molecules is responsible for the dark gas. This interpretation is supported by a recent finding of cool HI gas corresponding to the TeV $\gamma$-ray shell in the SNR RX J1713.7-3946 (Fukui et al. 2011). Such HI gas is not recognized under a usual assumption of optically thin HI emission but is identified by a careful analysis considering optically thick HI. The typical column density of such HI gas is a few times $10^{21} \mathrm{~cm}^{-2}$ and is also identified as visual extinction.
\end{abstract}

Keywords. ISM: clouds; cosmic rays; supernova remnants, gamma rays: observations, dark gas

Interstellar medium (ISM) consists of gas and dust and the gas is either in molecular or atomic form if neutral. The molecular gas typically has temperatures of $\sim 10 \mathrm{~K}$ and densities of $\sim 100-1000 \mathrm{~cm}^{-3}$, and is detected in the rotational transitions of $\mathrm{CO}$ and other molecules. On the other hand, the atomic gas has higher temperatures, $\sim 100 \mathrm{~K}$, and lower densities, $\sim 1 \mathrm{~cm}^{-3}$, and is detected in the $21 \mathrm{~cm}$ transition of HI. It is important to understand physical conditions of the neutral ISM quantitatively in order to elucidate the formation of stars and the interaction between cosmic rays (CR) and the ISM, since the ISM plays a major role in the evolution of the Galaxy.

It has been argued that there is yet an additional component in the ISM, so called "dark gas", which is not detectable either by CO or HI. The dark gas is suggested from $\gamma$-ray observations of EGRET as well as sub-mm observations by Planck (Grenier et al. 2005; Ade et al. 2011). CR protons interact with the ISM protons to produce neutral pions which decay into two $\gamma$-rays. The dark gas may provide a possible link between CR protons and the ISM. We here discuss the origin and implications of the dark gas in the light of a new study of the ISM protons in a TeV $\gamma$-ray SNR.

\section{Dark gas}

Grenier et al. (2005) compared the EGRET $\gamma$-ray data with CO and HI in order to test if the $\gamma$-rays show spatial correspondence with the neutral gas. The distribution of the $\gamma$-rays should correspond to the ISM proton distribution if the CR density distribution is uniform. Grenier et al. (2005) found that the $\gamma$-ray distribution shows generally a good correspondence with $\mathrm{CO}$ and $\mathrm{HI}$ whereas there is some residual $\gamma$-rays that has no 


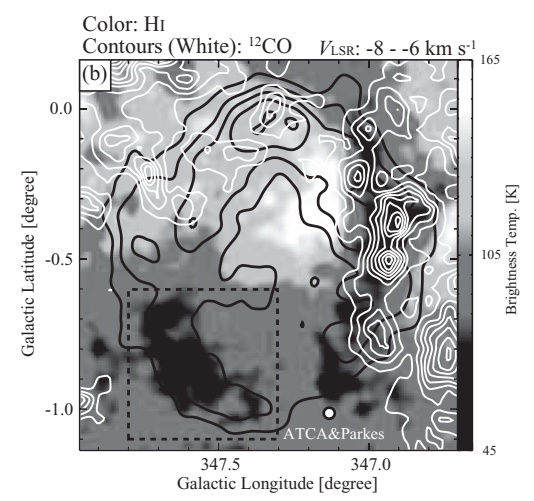

Figure 1. HI distribution towards RX J1713.7-3946 (grey scale). White and black contours show ${ }^{12} \mathrm{CO} J=1-0$ and $\mathrm{TeV} \gamma$-rays, respectively. Dotted box is the region shown in Figure 2(a).

correspondence either with CO or HI. The authors called this residual component "dark gas" and estimated that the dark gas has proton column densities $N_{\mathrm{p}}$ of $\sim 3 \times 10^{20}$ $3 \times 10^{21} \mathrm{~cm}^{-2}$ and masses of $20-100 \%$ of those estimated from CO and HI.

More recently, observations with the Planck satellite showed that the $\mathrm{mm}$ and submm dust emission also has a residual component not explicable either by CO or HI (Ade et al. 2011). The dark gas identified in the Planck data has mass $\sim 30 \%$ larger than those estimated from $\mathrm{CO}$ and $\mathrm{HI}$ in a column density range of $N_{\mathrm{p}} \sim 1-5 \times 10^{21} \mathrm{~cm}^{-2}$. These properties appear consistent with those of the dark gas derived from the EGRET data.

Some of the previous works discussed that lower density molecular gas without CO may correspond to the dark gas. Wolfire et al. (2010) studied a steady state chemistry model of a GMC and argued that GMCs may have molecular envelopes where CO is photo-dissociated. Such envelopes may explain the dark gas. It is however to be noted that the time scale of $\mathrm{H}_{2}$ formation on the dust grain surface is fairly long as $t=10^{7}$ year at a HI density of $100 \mathrm{~cm}^{-2}$ (e.g., Koyama \& Inutsuka 2000) and the evolutionary time scale of a GMC, 10 Myrs (e.g., Fukui and Kawamura 2010), may be only marginally long for $\mathrm{H}_{2}$ formation from $\mathrm{HI}$ (c.f., Wolfire et al. 2010). The dark gas is so far identified only for the local clouds at galactic latitudes higher than $10^{\circ}$ where only low-mass clouds are distributed (Grenier et al. 2005; Ade et al. 2011). Such molecular clouds have smaller evolutionary timescales less than $10^{6}$ yrs, too short to form $\mathrm{H}_{2}$ envelopes from $\mathrm{HI}$, and it may be unlikely that the molecular gas without $\mathrm{CO}$ corresponds to the dark gas.

\section{TeV $\gamma$-ray SNR RX J1713.7-3946}

We shall here discuss cool and dense atomic gas as an alternative candidate of the dark gas. A recent study of cool HI gas seen as self-absorption in the TeV $\gamma$-ray SNR RX J1713.7-3946 provides a hint to the alternative (Fukui et al. 2011). We shall summarize the main points of the work below from Fukui et al. (2011).

RX J1713.7-3946 is the brightest TeV $\gamma$-ray supernova remnant (SNR) (Aharonian et al. 2006). CO clouds interacting with the SNR were discovered in the $2.6 \mathrm{~mm} \mathrm{CO}$ emission (Fukui et al. 2003). Recently, Fukui et al. (2011) carried out a combined analysis of both the ${ }^{12} \mathrm{CO} J=1-0$ and $\mathrm{HI}$ and showed a good spatial correspondence with the TeV $\gamma$-rays. Figure 1 indicates that the HI distribution shows two dark regions; one of them in the west coincides with $\mathrm{CO}$ whereas the other in the southeast, the dark HI SE cloud, shows no CO emission. The dark HI SE cloud corresponds well with the TeV $\gamma$-ray shell and the authors interpreted that the HI has a broad self-absorption as shown in 

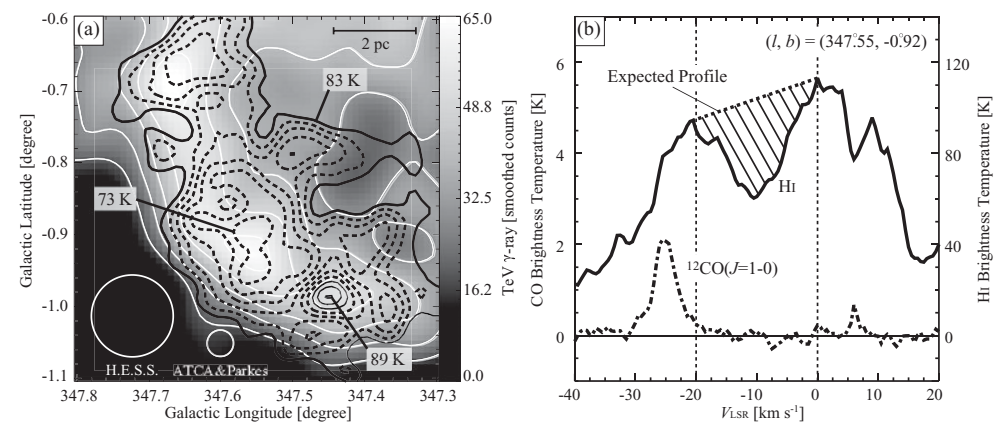

Figure 2. (a) The $\mathrm{TeV} \gamma$-rays and HI distributions toward the SE cloud. (b) Example spectra of HI and ${ }^{12} \mathrm{CO} J=1-0$ in the SE cloud. The shaded area shows an expected HI profile.
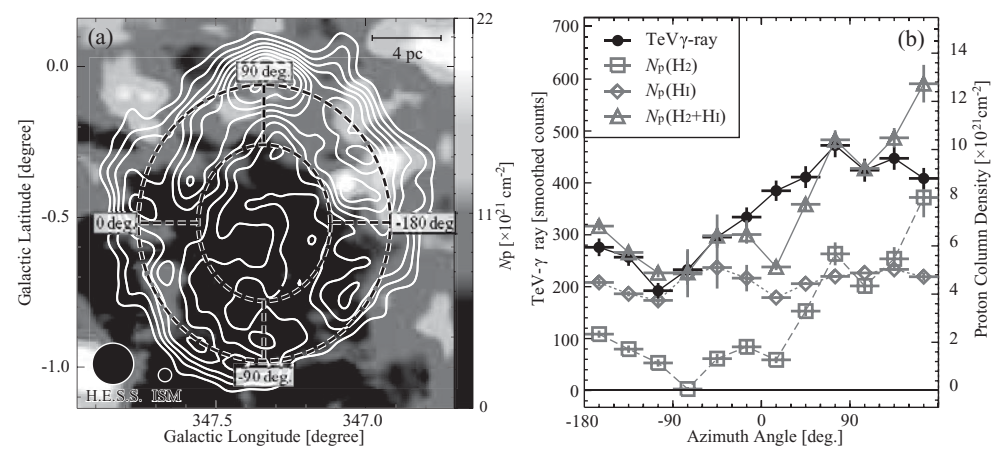

Figure 3. (a) Distributions of column density of the total ISM protons $N_{\mathrm{p}}\left(\mathrm{H}_{2}+\mathrm{HI}\right)$. Contours show the TeV $\gamma$-rays. (b) Azimuthal distributions of $N_{\mathrm{p}}\left(\mathrm{H}_{2}\right), N_{\mathrm{p}}(\mathrm{HI}), N_{\mathrm{p}}\left(\mathrm{H}_{2}+\mathrm{HI}\right)$, and $\mathrm{TeV}$ $\gamma$-ray-smoothed counts per beam in the ring shown in the figure (a).

Figure 2. The visual extinction is also enhanced along the shell in the SE cloud, lending another support for the self-absorption interpretation.

Figures 3(a) shows the distribution of the total ISM protons. We find it corresponds well to the $\gamma$-rays spatially. The azimuthal distribution of the $\gamma$-rays and the total ISM protons shown in Figure 3(b) also shows the correspondence; in particular, the dark HI $\mathrm{SE}$ cloud makes the correspondence significantly better than in the case of $\mathrm{H}_{2}$ alone.

The observed dark gas properties are summarized as follows; spin temperature $T_{\mathrm{S}}$, column density $N_{\mathrm{p}}(\mathrm{HI})$ and optical depth $\tau$ are $\sim 40 \mathrm{~K}, \sim 5 \times 10^{21} \mathrm{~cm}^{-2}$ and $0.7-1.4$, respectively. These characteristics are consistent with those of Planck and EGRET. If we assume the optically thin HI in the dark HI SE cloud, we have an estimate of HI column density $\sim 30-50 \%$ less than the self-absorption case. We therefore suggest that such cool and dense HI gas is ubiquitous and may be responsible for the dark gas.

\section{Cool and dense HI gas as the dark gas candidate}

We shall discuss general properties of the cool and dense HI gas as the candidate of the dark gas. The formation of such HI gas is naturally explained by compression due to stellar winds of high mass stars in a typical time scale of 1 Myr (e.g., Yamamoto et al. 2006). The equation 3.1 is used to estimate the column density of $\mathrm{HI}, N_{\mathrm{p}}(\mathrm{HI})$.

$$
N_{\mathrm{p}}(\mathrm{HI})=\frac{32 \pi \nu k_{\mathrm{b}}}{3 c^{3} h A_{\mathrm{ul}}} \int_{\nu_{1}}^{\nu_{2}} \Delta T_{\mathrm{b}} d \nu=1.823 \times 10^{18} \times W(\mathrm{HI})\left(\mathrm{cm}^{-2}\right),
$$




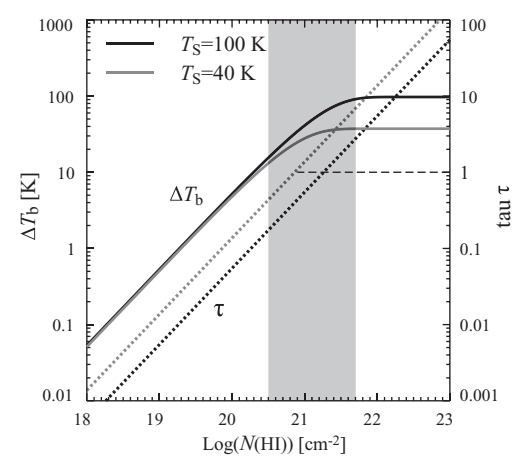

Figure 4. $\Delta T_{\mathrm{b}}$ and $\tau$ variations depending on $N_{\mathrm{p}}(\mathrm{HI})$. Here $\Delta v$ of $10 \mathrm{~km} \mathrm{~s}^{-1}$ is assumed. The filled area shows a $N_{\mathrm{p}}$ range of the dark gas (Grenier et al. 2005; Ade et al. 2011).

where $\Delta T_{\mathrm{b}}$ and $W(\mathrm{HI})$ is the brightness temperature and integrated intensity of $\mathrm{HI}$, respectively. Here optically thin HI is assumed. It usually works well, but we have to consider optical depth $\tau$ and spin temperature $T_{\mathrm{s}}$ in the cool and dense $\mathrm{HI}$ as follows,

$$
\tau=\frac{1}{1.823 \times 10^{18}} \frac{1}{T_{\mathrm{s}}(\mathrm{K})} \frac{N_{\mathrm{p}}(\mathrm{HI})\left(\mathrm{cm}^{-2}\right)}{\Delta v\left(\mathrm{~km} \mathrm{~s}^{-1}\right)},
$$

where $\Delta v$ is the velocity width of HI profile. The brightness temperature $\Delta T_{\mathrm{b}}$ is described as $\Delta T_{\mathrm{b}}=\left(T_{\mathrm{s}}-T_{\mathrm{bg}}\right)(1-\exp (-\tau))$ where $T_{\mathrm{bg}}$ is temperature of background radiation field.

Figure 4 shows variations of $\Delta T_{\mathrm{b}}$ and $\tau$ depending on $N_{\mathrm{p}}(\mathrm{HI}) . \Delta T_{\mathrm{b}}$ becomes saturated at $N_{\mathrm{p}}(\mathrm{HI})$ of more than $10^{21} \mathrm{~cm}^{-2}$ and at $\tau$ of more than $\sim 1.0$. For such a moderately optically thick condition, we underestimate the column density $N_{\mathrm{p}}(\mathrm{HI})$ in the optically thin assumption (see Eq. 3.1). The filled area in the figure shows the estimated column densities $N_{\mathrm{p}}$ of the dark gas obtained with the Planck and EGRET results, which correspond well to the regime where $\Delta T_{\mathrm{b}}$ becomes saturated.

The spin temperature $T_{\mathrm{s}}$ of $\mathrm{HI}$ is $\sim 100 \mathrm{~K}$ or higher in warm neutral medium and decreases with density from $100 \mathrm{~K}$ to $10 \mathrm{~K}$ in a range of $N_{\mathrm{p}} \sim 10^{20}-10^{21} \mathrm{~cm}^{-2}$ because of higher shielding of stellar photons and enhanced cooling with density increase. (e.g., Figure 2 in Goldsmith et al. 2007). Because the cooling timescale is much shorter than the $\mathrm{H}_{2}$ formation timescale (e.g., Koyama \& Inutsuka 2000), the cool and dense atomic gas of $100 \mathrm{~cm}^{-3}$ can stay atomic without forming molecules for $\sim 10^{7}$ years.

We summarize that the present work has shown cool and dense HI gas is a viable candidate for the dark gas which is not explicable either by $\mathrm{CO}$ or by the optically thin HI analysis. Further detailed properties of the cool and dense HI remains to be explored.

\section{References}

Ade, P. A. R. et al. 2011, arXiv: 1101.2029v1

Aharonian, F. A., et al. 2006, A\&BA, 449, 223

Goldsmith, P. F., Li, D., \& Krčo, M. 2007, ApJ, 654, 273

Grenier, I. A., Casandjian, J.-M., \& Terrier, R. 2005, Science, 307, 1292

Fukui, Y., et al. 2003, PASJ, 55, 61

Fukui, Y., et al. 2011, ApJ, in press

Fukui, Y. \& Kawamura, A. 2010, ARAA, 48, 547

Koyama, H. \& Inutsuka, S. 2000, ApJ, 532, 980

McClure-Griffiths, N. M. et al. 2005, ApJS, 158, 178

Sato, F. \& Fukui, Y. 1978, ApJ, 83, 1607

Yamamoto et al. 2006, ApJ, 642, 307 